\title{
Applications of Arithmetic Geometric Mean Inequality
}

\author{
Wasim Audeh \\ Department of Mathematics, University of Petra, Amman, Jordan \\ Email:waudeh@uop.edu.jo
}

How to cite this paper: Audeh, W. (2017) Applications of Arithmetic Geometric Mean Inequality. Advances in Linear Algebra \& Matrix Theory, 7, 29-36.

https://doi.org/10.4236/alamt.2017.72004

Received: March 26, 2017

Accepted: June 18, 2017

Published: June 21, 2017

Copyright (C) 2017 by author and Scientific Research Publishing Inc. This work is licensed under the Creative Commons Attribution International License (CC BY 4.0).

http://creativecommons.org/licenses/by/4.0/

\begin{abstract}
The well-known arithmetic-geometric mean inequality for singular values, due to Bhatia and Kittaneh, is one of the most important singular value inequalities for compact operators. The purpose of this study is to give new singular value inequalities for compact operators and prove that these inequalities are equivalent to arithmetic-geometric mean inequality, the way by which several future studies could be done.
\end{abstract}

\section{Keywords}

Compact Operator, Inequality, Positive Operator, Singular Value

\section{Fundamental Principles}

Let $B(H)$ indicate the set of all bounded linear operators on a complex separable Hilbert space $H$, and let $K(H)$ indicate the two-sided ideal of compact operators in $B(H)$. If $T \in K(H)$, the singular values of $T$, denoted by $s_{1}(T), s_{2}(T), \cdots$ are the eigenvalues of the positive operator $|T|=\left(T^{*} T\right)^{1 / 2}$ ordered as $s_{1}(T) \geq s_{2}(T) \geq \cdots$ and repeated according to multiplicity. It is well known that $s_{j}(T)=s_{j}\left(T^{*}\right)=s_{j}(|T|)$ for $j=1,2, \cdots$. It follows by Weyl's monotonicity principle (see, e.g., [1], p. 63 or [2], p. 26) that if

$S, T \in K(H)$ are positive and $S \leq T$, then $s_{j}(S) \leq s_{j}(T)$ for $j=1,2, \cdots$. Moreover, for $S, T \in K(H), s_{j}(S) \leq s_{j}(T)$ if and only if

$s_{j}(S \oplus S) \leq s_{j}(T \oplus T)$ for $j=1,2, \cdots$. Here, we use the direct sum notation $S \oplus T$ for the block-diagonal operator $\left[\begin{array}{ll}S & 0 \\ 0 & T\end{array}\right]$ defined on $H \oplus H$. The singular values of $S \oplus T$ and $\left[\begin{array}{ll}0 & T \\ S & 0\end{array}\right]$ are the same, and they consist of those of $S$ together with those of $T$.

Bhatia and Kittaneh have proved in [3] that if $A, B \in K(H)$ such that $A$ is 
self-adjoint, $B \geq 0$, and $\pm A \leq B$, then

$$
s_{j}(A) \leq s_{j}(B \oplus B)
$$

for $j=1,2, \cdots$.

Audeh and Kittaneh in [4] prove inequality which is equivalent to inequality (1.1):

If $A, B, C \in K(H)$ such that $\left[\begin{array}{cc}A & B \\ B^{*} & C\end{array}\right] \geq 0$, then

$$
s_{j}(B) \leq s_{j}(A \oplus C)
$$

for $j=1,2, \cdots$.

The well-known arithmetic-geometric mean inequality for singular values, due to Bhatia and Kittaneh [5], says that if $A, B \in K(H)$, then

$$
2 s_{j}\left(A B^{*}\right) \leq s_{j}\left(A^{*} A+B^{*} B\right)
$$

for $j=1,2, \cdots$. On the other hand, Zhan has proved in [6] that if $A, B \in K(H)$ are positive, then

$$
s_{j}(A-B) \leq s_{j}(A \oplus B)
$$

for $j=1,2, \cdots$. Moreover, Tao has proved in [7] that if $A, B, C \in K(H)$ such that $\left[\begin{array}{cc}A & B \\ B^{*} & C\end{array}\right] \geq 0$, then

$$
2 s_{j}(B) \leq s_{j}\left[\begin{array}{cc}
A & B \\
B^{*} & C
\end{array}\right]
$$

for $j=1,2, \cdots$.

Audeh and Kittaneh have proved in [4] that:

If $A, B \in K(H)$ such that $A$ is self-adjoint, $B \geq 0$, and $\pm A \leq B$, then

$$
2 s_{j}(A) \leq s_{j}((B+A) \oplus(B-A))
$$

for $j=1,2, \cdots$.

It has been pointed out in [4] that the four inequalities (1.3)-(1.6) are equivalent.

Moreover, Tao in [7] uses inequality (1.3) to prove that if $A$ and $B$ are positive operators in $K(H), r \geq 0$. Then

$$
2 s_{j}\left(A^{1 / 2}(A+B)^{r} B^{1 / 2}\right) \leq s_{j}(A+B)^{r+1}
$$

for $j=1,2, \cdots$.

\section{Introduction}

In this study, we will present several new inequalities, and prove that they are equivalent to arithmetic-geometric mean inequality.

The following are the proved inequalities in this study:

Let $A, C$ and $D$ be operators in $K(H)$ where $A \geq 0, C$ and $D$ arbitrary operators. Then

$$
2 s_{j}\left(C A D^{*}\right) \leq s_{j}\left[\begin{array}{ll}
C A C^{*} & C A D^{*} \\
D A C^{*} & D A D^{*}
\end{array}\right]
$$


for $j=1,2, \cdots$.

Let $C, D$ and $X$ be arbitrary operators in $K(H)$. Then we have

$$
2 s_{j}\left(D X C^{*}\right) \leq s_{j}\left[\begin{array}{cc}
D\left|X^{*}\right| D^{*} & D X C^{*} \\
C X^{*} D^{*} & C|X| C^{*}
\end{array}\right]
$$

for $j=1,2, \cdots$.

Let $A_{1}, A_{2}, A_{3}, A_{4}$ be operators in $K(H)$. Then

$$
2 s_{j}\left[\begin{array}{ll}
A_{1} A_{2}^{*} & A_{1} A_{4}^{*} \\
A_{3} A_{2}^{*} & A_{3} A_{4}^{*}
\end{array}\right] \leq s_{j}\left(\left|A_{1}\right|^{2}+\left|A_{2}\right|^{2}+\left|A_{3}\right|^{2}+\left|A_{4}\right|^{2}\right)
$$

for $j=1,2, \cdots$.

If $A, B, C, D$ and $X$ are operators in $K(H)$. Then

$$
\begin{aligned}
& 2 s_{j}\left(A|X| C^{*} \pm B X C^{*} \pm A X^{*} D^{*}+B\left|X^{*}\right| D^{*}\right) \\
& \leq s_{j}\left[\begin{array}{ll}
A|X| A^{*} \pm B X A^{*} \pm A X^{*} B^{*}+B\left|X^{*}\right| B^{*} & A|X| C^{*} \pm B X C^{*} \pm A X^{*} D^{*}+B\left|X^{*}\right| D^{*} \\
C|X| A^{*} \pm D X A^{*} \pm C X^{*} B^{*}+D\left|X^{*}\right| B^{*} & C|X| C^{*} \pm D X C^{*} \pm C X^{*} D^{*}+D\left|X^{*}\right| D^{*}
\end{array}\right] \\
& \quad \text { for } j=1,2, \cdots .
\end{aligned}
$$

Let $A_{1}, A_{2}, \cdots, A_{n}$ be positive operators in $K(H), r \geq 0$. Then

$$
\begin{aligned}
& 2 s_{j}\left[A_{1}^{1 / 2}\left(A_{1}+A_{n}\right)^{r} A_{n}^{1 / 2} \oplus A_{2}^{1 / 2}\left(A_{2}+A_{n-1}\right)^{r} A_{n-1}^{1 / 2} \oplus \cdots \oplus A_{n}^{1 / 2}\left(A_{n}+A_{1}\right)^{r} A_{1}^{1 / 2}\right] \\
& \leq s_{j}\left[\left(A_{1}+A_{n}\right)^{r+1} \oplus\left(A_{2}+A_{n-1}\right)^{r+1} \oplus \cdots \oplus\left(A_{n}+A_{1}\right)^{r+1}\right]
\end{aligned}
$$

for $j=1,2, \cdots$.

\section{Main Results}

Our first singular value inequality needs the following lemma.

Lemma 1: Let $A$ be a positive operator in $K(H), X$ be an arbitrary operator in $K(H)$. Then we have

$$
X A X^{*} \geq 0
$$

Now we will prove the first Theorem which is equivalent to arithmeticgeometric mean inequality.

Theorem 3.1 Let $A, C$ and $D$ be operators in $K(H)$ where $A \geq 0, C$ and $D$ arbitraryoperators. Then

$$
2 s_{j}\left(C A D^{*}\right) \leq s_{j}\left[\begin{array}{ll}
C A C^{*} & C A D^{*} \\
D A C^{*} & D A D^{*}
\end{array}\right]
$$

for $j=1,2, \cdots$.

Proof. Let $X=\left[\begin{array}{cc}A & 0 \\ 0 & A\end{array}\right] \geq 0$ (because $A \geq 0$ by assumption), and let $M=\left[\begin{array}{ll}C & 0 \\ D & 0\end{array}\right]$. Then we have

$$
M X M^{*}=\left[\begin{array}{cc}
C & 0 \\
D & 0
\end{array}\right]\left[\begin{array}{cc}
A & 0 \\
0 & A
\end{array}\right]\left[\begin{array}{cc}
C^{*} & D^{*} \\
0 & 0
\end{array}\right]=\left[\begin{array}{cc}
C A C^{*} & C A D^{*} \\
D A C^{*} & D A D^{*}
\end{array}\right] \geq 0
$$


From (1.5) we have

$$
2 s_{j}\left(C A D^{*}\right) \leq s_{j}\left[\begin{array}{ll}
C A C^{*} & C A D^{*} \\
D A C^{*} & D A D^{*}
\end{array}\right]
$$

for $j=1,2, \cdots$.

Now we will prove that Theorem (3.1) is equivalent to arithmetic-geometric mean inequality.

Theorem 3.2 The following statements are equivalent:

1) Let $X, Y \in K(H)$, then

$$
2 s_{j}\left(X Y^{*}\right) \leq s_{j}\left(X^{*} X+Y^{*} Y\right)
$$

for $j=1,2, \cdots$.

2) Let $A, C$ and $D$ be operators in $K(H)$ where $A \geq 0, C$ and $D$ arbitrary operators. Then

$$
2 s_{j}\left(C A D^{*}\right) \leq s_{j}\left[\begin{array}{ll}
C A C^{*} & C A D^{*} \\
D A C^{*} & D A D^{*}
\end{array}\right]
$$

for $j=1,2, \cdots$.

Proof. 1) $\rightarrow$ 2) Let $X=C A^{1 / 2}, Y=D A^{1 / 2}$.

Now apply arithmetic-geometric mean inequality to get

$$
2 s_{j}\left(C A^{1 / 2} A^{1 / 2} D^{*}\right) \leq s_{j}\left(A^{1 / 2} C^{*} C A^{1 / 2}+A^{1 / 2} Y^{*} Y A^{1 / 2}\right)
$$

for $j=1,2, \cdots$. But

$$
\begin{aligned}
s_{j}\left(A^{1 / 2} C^{*} C A^{1 / 2}+A^{1 / 2} Y^{*} Y A^{1 / 2}\right) & =s_{j}\left[\begin{array}{cc}
A^{1 / 2} C^{*} & A^{1 / 2} D^{*} \\
0 & 0
\end{array}\right]\left[\begin{array}{cc}
C A^{1 / 2} & 0 \\
D A^{1 / 2} & 0
\end{array}\right] \\
& =s_{j}\left[\begin{array}{cc}
C A^{1 / 2} & 0 \\
D A^{1 / 2} & 0
\end{array}\right]\left[\begin{array}{cc}
A^{1 / 2} C^{*} & A^{1 / 2} D^{*} \\
0 & 0
\end{array}\right] \\
& =s_{j}\left[\begin{array}{cc}
C A C^{*} & C A D^{*} \\
D A C^{*} & D A D^{*}
\end{array}\right] .
\end{aligned}
$$

The above steps implies that

$$
2 s_{j}\left(C A D^{*}\right) \leq s_{j}\left[\begin{array}{cc}
C A C^{*} & C A D^{*} \\
D A C^{*} & D A D^{*}
\end{array}\right] \text { for } j=1,2, \cdots .
$$

$2) \rightarrow 1$ ) The matrix $\left[\begin{array}{cc}C A C^{*} & C A D^{*} \\ D A C^{*} & D A D^{*}\end{array}\right]$ can be factorized as

$$
\begin{aligned}
& {\left[\begin{array}{cc}
C A^{1 / 2} & 0 \\
D A^{1 / 2} & 0
\end{array}\right]\left[\begin{array}{cc}
A^{1 / 2} C^{*} & A^{1 / 2} D^{*} \\
0 & 0
\end{array}\right], \text { but it is well known that } } \\
& s_{j}(T)=s_{j}\left(T^{*}\right)=s_{j}(|T|) \text { for } j=1,2, \cdots \text {. So } \\
& s_{j}\left[\begin{array}{cc}
C A C^{*} & C A D^{*} \\
D A C^{*} & D A D^{*}
\end{array}\right]=s_{j}\left[\begin{array}{cc}
C A^{1 / 2} & 0 \\
D A^{1 / 2} & 0
\end{array}\right]\left[\begin{array}{cc}
A^{1 / 2} C^{*} & A^{1 / 2} D^{*} \\
0 & 0
\end{array}\right] \\
&=s_{j}\left[\begin{array}{cc}
A^{1 / 2} C^{*} & A^{1 / 2} D^{*} \\
0 & 0
\end{array}\right]\left[\begin{array}{cc}
C A^{1 / 2} & 0 \\
D A^{1 / 2} & 0
\end{array}\right] \\
&=s_{j}\left(A^{1 / 2} C^{*} C A^{1 / 2}+A^{1 / 2} D^{*} D A^{1 / 2}\right)
\end{aligned}
$$


for $j=1,2, \cdots$, from (2) we have

$$
2 s_{j}\left(C A D^{*}\right) \leq s_{j}\left(A^{1 / 2} C^{*} C A^{1 / 2}+A^{1 / 2} D^{*} D A^{1 / 2}\right)
$$

for $j=1,2, \cdots$. Now let $A=I$ in Inequality (3.2) we get

$$
2 s_{j}\left(C D^{*}\right) \leq s_{j}\left(C^{*} C+D^{*} D\right)
$$

for $j=1,2, \cdots$, which is the arithmetic-geometric mean inequality.

The following lemma which was proved by Bhatia [1] is essential to prove the next theorem.

Lemma 2 Let $X$ be arbitrary operator in $B(H)$. Then

$$
\left[\begin{array}{cc}
|X| & \pm X^{*} \\
\pm X & \left|X^{*}\right|
\end{array}\right] \geq 0
$$

Now we will prove the following theorem which is more general than Theorem (3.1) and equivalent to arithmetic-geometric mean inequality.

Theorem 3.3 Let $C, D$ and $X$ be arbitrary operators in $K(H)$. Then we have

$$
2 s_{j}\left(D X C^{*}\right) \leq s_{j}\left[\begin{array}{cc}
D\left|X^{*}\right| D^{*} & D X C^{*} \\
C X^{*} D^{*} & C|X| C^{*}
\end{array}\right]
$$

for $j=1,2, \cdots$.

Proof. Applying Lemma (2) gives $A=\left[\begin{array}{cc}|X| & X^{*} \\ X & \left|X^{*}\right|\end{array}\right] \geq 0$ for an arbitrary operator $X$. Let $N=\left[\begin{array}{ll}0 & D \\ C & 0\end{array}\right]$, by using Inequality (3.1) we have $N A N^{*}=\left[\begin{array}{cc}D\left|X^{*}\right| D^{*} & D X C^{*} \\ C X^{*} D^{*} & C|X| C^{*}\end{array}\right] \geq 0$. Hence using Inequality (1.5) gives $2 s_{j}\left(D X C^{*}\right) \leq s_{j}\left[\begin{array}{cc}D\left|X^{*}\right| D^{*} & D X C^{*} \\ C X^{*} D^{*} & C|X| C^{*}\end{array}\right]$.

Remark 1 Theorem (3.3) is generalization of Theorem (3.1) because here $X$ is arbitrary operator but there $A$ should be positive operator.

Remark 2 Inequality (2.2) is equivalent to arithmetic-geometric mean inequality. We can prove this equivalent by similar steps used to prove Theorem (3.2).

The following theorem is a generalization of Theorem (3.1) and Theorem (3.3).

Theorem 3.4 Let $A, B, C, D$ and $X$ be arbitraryoperators in $K(H)$. Then we have

$$
\begin{aligned}
& 2 s_{j}\left(A|X| C^{*} \pm B X C^{*} \pm A X^{*} D^{*}+B\left|X^{*}\right| D^{*}\right) \\
& \leq s_{j}\left[\begin{array}{ll}
A|X| A^{*} \pm B X A^{*} \pm A X^{*} B^{*}+B\left|X^{*}\right| B^{*} & A|X| C^{*} \pm B X C^{*} \pm A X^{*} D^{*}+B\left|X^{*}\right| D^{*} \\
C|X| A^{*} \pm D X A^{*} \pm C X^{*} B^{*}+D\left|X^{*}\right| B^{*} & C|X| C^{*} \pm D X C^{*} \pm C X^{*} D^{*}+D\left|X^{*}\right| D^{*}
\end{array}\right]
\end{aligned}
$$




$$
\begin{aligned}
& \text { for } j=1,2, \cdots \text {. } \\
& \text { Proof. Let } M=\left[\begin{array}{cc}
A & B \\
C & D
\end{array}\right], Z=\left[\begin{array}{cc}
|X| & \pm X^{*} \\
\pm X & \left|X^{*}\right|
\end{array}\right] \text {. Then } \\
& M Z M^{*}=\left[\begin{array}{cc}
A & B \\
C & D
\end{array}\right]\left[\begin{array}{cc}
|X| & \pm X^{*} \\
\pm X & \left|X^{*}\right|
\end{array}\right]\left[\begin{array}{cc}
A^{*} & C^{*} \\
B^{*} & D^{*}
\end{array}\right] \geq 0 \text {. Hence } \\
& {\left[\begin{array}{ll}
A|X| A^{*} \pm B X A^{*} \pm A X^{*} B^{*}+B\left|X^{*}\right| B^{*} & A|X| C^{*} \pm B X C^{*} \pm A X^{*} D^{*}+B\left|X^{*}\right| D^{*} \\
C|X| A^{*} \pm D X A^{*} \pm C X^{*} B^{*}+D\left|X^{*}\right| B^{*} & C|X| C^{*} \pm D X C^{*} \pm C X^{*} D^{*}+D\left|X^{*}\right| D^{*}
\end{array}\right] \geq 0,}
\end{aligned}
$$

use Inequality (1.5) to get the required result.

Remark 3 Replace B, D by 0 in Inequality (2.4) will gives Inequality (2.1).

Remark 4 Replace $A$, C by 0 in Inequality (2.4) will also gives Inequality (2.1).

Now we will use Inequality (1.3) to prove the following theorem, then we will show that they are equivalent.

Theorem 3.5 Let $A_{1}, A_{2}, A_{3}, A_{4}$ be operators in $K(H)$. Then

$$
2 s_{j}\left[\begin{array}{ll}
A_{1} A_{2}^{*} & A_{1} A_{4}^{*} \\
A_{3} A_{2}^{*} & A_{3} A_{4}^{*}
\end{array}\right] \leq s_{j}\left(\left|A_{1}\right|^{2}+\left|A_{2}\right|^{2}+\left|A_{3}\right|^{2}+\left|A_{4}\right|^{2}\right)
$$

for $j=1,2, \cdots$.

Proof. Let $A=\left[\begin{array}{ll}A_{1} & 0 \\ A_{3} & 0\end{array}\right], B=\left[\begin{array}{ll}A_{2} & 0 \\ A_{4} & 0\end{array}\right]$. Then $A B^{*}=\left[\begin{array}{ll}A_{1} A_{2}^{*} & A_{1} A_{4}^{*} \\ A_{3} A_{2}^{*} & A_{3} A_{4}^{*}\end{array}\right]$, and $A^{*} A+B^{*} B=\left|A_{1}\right|^{2}+\left|A_{2}\right|^{2}+\left|A_{3}\right|^{2}+\left|A_{4}\right|^{2}$. Now use Inequality (1.3) we get

$$
2 s_{j}\left[\begin{array}{ll}
A_{1} A_{2}^{*} & A_{1} A_{4}^{*} \\
A_{3} A_{2}^{*} & A_{3} A_{4}^{*}
\end{array}\right] \leq s_{j}\left(\left|A_{1}\right|^{2}+\left|A_{2}\right|^{2}+\left|A_{3}\right|^{2}+\left|A_{4}\right|^{2}\right)
$$

for $j=1,2, \cdots$.

Now we will prove that Inequality (2.3) is equivalent to Inequality (1.3).

Theorem 3.6 The following statements are equivalent.

1) Let $A, B \in K(H)$. Then

$$
2 s_{j}\left(A B^{*}\right) \leq s_{j}\left(A^{*} A+B^{*} B\right)
$$

for $j=1,2, \cdots$.

2) Let $A_{1}, A_{2}, A_{3}, A_{4}$ be operators in $K(H)$. Then

$$
2 s_{j}\left[\begin{array}{ll}
A_{1} A_{2}^{*} & A_{1} A_{4}^{*} \\
A_{3} A_{2}^{*} & A_{3} A_{4}^{*}
\end{array}\right] \leq s_{j}\left(\left|A_{1}\right|^{2}+\left|A_{2}\right|^{2}+\left|A_{3}\right|^{2}+\left|A_{4}\right|^{2}\right)
$$

for $j=1,2, \cdots$.

Proof. 1) $\rightarrow$ 2) It is the proof of Theorem (3.5).

2) $\rightarrow$ 1) By replacing $A_{2}=A_{4}=B$ and $A_{1}=A_{3}=A$ in Inequality (2.3), we get $2 s_{j}\left[\begin{array}{ll}A B^{*} & A B^{*} \\ A B^{*} & A B^{*}\end{array}\right] \leq s_{j}\left(|A|^{2}+|B|^{2}+|A|^{2}+|B|^{2}\right)$. From this we reach to $4 s_{j}\left(A B^{*}\right) \leq 2 s_{j}\left(A^{*} A+B^{*} B\right)$ which implies that $2 s_{j}\left(A B^{*}\right) \leq s_{j}\left(A^{*} A+B^{*} B\right)$ for $j=1,2, \cdots$.

In the rest of this paper, we will prove new inequality which is equivalent to Inequality (1.7). 
Theorem 3.7 Let $A_{1}, A_{2}, \cdots, A_{n}$ be positive operators in $K(H), n$ is an even integer, $r \geq 0$. Then

$$
\begin{aligned}
& 2 s_{j}\left[A_{1}^{1 / 2}\left(A_{1}+A_{n}\right)^{r} A_{n}^{1 / 2} \oplus A_{2}^{1 / 2}\left(A_{2}+A_{n-1}\right)^{r} A_{n-1}^{1 / 2} \oplus \cdots \oplus A_{n}^{1 / 2}\left(A_{n}+A_{1}\right)^{r} A_{1}^{1 / 2}\right] \\
& \leq s_{j}\left[\left(A_{1}+A_{n}\right)^{r+1} \oplus\left(A_{2}+A_{n-1}\right)^{r+1} \oplus \cdots \oplus\left(A_{n}+A_{1}\right)^{r+1}\right]
\end{aligned}
$$

for $j=1,2, \cdots$.

$$
\begin{aligned}
& \text { Proof. Let } C=\left[\begin{array}{cccc}
A_{1} & 0 & \cdots & 0 \\
0 & A_{2} & \cdots & 0 \\
0 & 0 & \ddots & \vdots \\
0 & 0 & \cdots & A_{n}
\end{array}\right], D=\left[\begin{array}{cccc}
A_{n} & 0 & \cdots & 0 \\
0 & A_{n-1} & \cdots & 0 \\
0 & 0 & \ddots & \vdots \\
0 & 0 & \cdots & A_{1}
\end{array}\right] \text {. Then we have } \\
& C^{1 / 2}(C+D)^{r} D^{1 / 2}=\left[\begin{array}{cccc}
A_{1}^{1 / 2}\left(A_{1}+A_{n}\right)^{r} A_{n}^{1 / 2} & 0 & \cdots & 0 \\
0 & A_{2}^{1 / 2}\left(A_{2}+A_{n-1}\right)^{r} A_{n-1}^{1 / 2} & \cdots & 0 \\
0 & 0 & \ddots & \vdots \\
0 & 0 & \cdots & A_{n}^{1 / 2}\left(A_{n}+A_{1}\right)^{r} A_{1}^{1 / 2}
\end{array}\right] \text {, } \\
& \text { and }(C+D)^{r+1}=\left[\begin{array}{cccc}
\left(A_{1}+A_{n}\right)^{r+1} & 0 & \cdots & 0 \\
0 & \left(A_{2}+A_{n-1}\right)^{r+1} & \cdots & 0 \\
0 & 0 & \ddots & \vdots \\
0 & 0 & \cdots & \left(A_{n}+A_{1}\right)^{r+1}
\end{array}\right] \text {. Now apply }
\end{aligned}
$$

Inequality (1.7) we get the result.

We will prove that Inequality (1.7) is equivalent to Inequality (3.5).

\section{Theorem 3.8 The following statements are equivalent.}

1) Let $A$ and $B$ be positive operators in $K(H), r \geq 0$. Then

$$
2 s_{j}\left(A^{1 / 2}(A+B)^{r} B^{1 / 2}\right) \leq s_{j}(A+B)^{r+1}
$$

for $j=1,2, \cdots$.

2) Let $A_{1}, A_{2}, \cdots, A_{n}$ be positive operators in $K(H), n$ is even integer, $r \geq 0$. Then

$$
\begin{aligned}
& 2 s_{j}\left[A_{1}^{1 / 2}\left(A_{1}+A_{n}\right)^{r} A_{n}^{1 / 2} \oplus A_{2}^{1 / 2}\left(A_{2}+A_{n-1}\right)^{r} A_{n-1}^{1 / 2} \oplus \cdots \oplus A_{n}^{1 / 2}\left(A_{n}+A_{1}\right)^{r} A_{1}^{1 / 2}\right] \\
& \leq s_{j}\left[\left(A_{1}+A_{n}\right)^{r+1} \oplus\left(A_{2}+A_{n-1}\right)^{r+1} \oplus \cdots \oplus\left(A_{n}+A_{1}\right)^{r+1}\right]
\end{aligned}
$$

for $j=1,2, \cdots$.

Proof. 1) $\rightarrow$ 2) This implication follows from the proof of Theorem 3.7.

$2) \rightarrow 1$ ) Let $A_{2}=A_{3}=\cdots=A_{n-1}=0$ in Inequality (3.5) to get

$$
2 s_{j}\left[A_{1}^{1 / 2}\left(A_{1}+A_{n}\right)^{r} A_{n}^{1 / 2} \oplus A_{n}^{1 / 2}\left(A_{n}+A_{1}\right)^{r} A_{1}^{1 / 2}\right] \leq s_{j}\left[\left(A_{1}+A_{n}\right)^{r+1} \oplus\left(A_{n}+A_{1}\right)^{r+1}\right]
$$

for $j=1,2, \cdots$. But $s_{j}\left(X^{*}\right)=s_{j}(X)$ and $s_{j}(X \oplus X) \leq s_{j}(Y \oplus Y)$ for $j=1,2, \cdots$.

If and only if $s_{j}(X) \leq s_{j}(Y)$, this gives

$$
2 s_{j}\left[A_{1}^{1 / 2}\left(A_{1}+A_{n}\right)^{r} A_{n}^{1 / 2}\right] \leq s_{j}\left(A_{1}+A_{n}\right)^{r+1}
$$

for $j=1,2, \cdots$, replace $A_{1}$ by $A, A_{n}$ by $B$ in this inequality we will get 


$$
2 s_{j}\left(A^{1 / 2}(A+B)^{r} B^{1 / 2}\right) \leq s_{j}(A+B)^{r+1}
$$

for $j=1,2, \cdots$.

\section{Conclusion}

Since this study has been completed, we can conclude that several singular value inequalities for compact operators are equivalent to arithmetic-geometric mean inequality, which in turns have many crucial applications in operator theory, and from this point we advise interested authors to join these results with results in other studies to make connection between several branches in operator theory.

\section{Acknowledgements}

The author is grateful to the University of Petra for its Support. The Author is grateful to the referee for his comments and suggestions.

\section{References}

[1] Bhatia, R. (1997) Matrix Analysis, GTM169. Springer-Verlag, New York. https://doi.org/10.1007/978-1-4612-0653-8

[2] Gohberg, I.C. and Krein, M.G. (1969) Introduction to the Theory of Linear Nonselfadjoint Operators. American Mathematical Society, Providence.

[3] Bhatia, R. and Kittaneh, F. (2008) The Matrix Arithmetic-Geometric Mean Inequality Revisited. Linear Algebra and Its Applications, 428, 2177-2191. https://doi.org/10.1016/j.laa.2007.11.030

[4] Audeh, W. and Kittaneh, F. (2012) Singular Value Inequalities for Compact Operators. Linear Algebra and Its Applications, 437, 2516-2522. https://doi.org/10.1016/j.laa.2012.06.032

[5] Bhatia, R. and Kittaneh, F. (1990) On the Singular Values of a Product of Operators. SIAM Journal on Matrix Analysis and Applications, 11, 272-277. https://doi.org/10.1137/0611018

[6] Zhan, X. (2000) Singular Values of Differences of Positive Semidefinite Matrices. SIAM Journal on Matrix Analysis and Applications, 22, 819-823. https://doi.org/10.1137/S0895479800369840

[7] Tao, Y. (2006) More Results on Singular Value Inequalities of Matrices. Linear Algebra and Its Applications, 416, 724-729. https://doi.org/10.1016/j.laa.2005.12.017 
Submit or recommend next manuscript to SCIRP and we will provide best service for you:

Accepting pre-submission inquiries through Email, Facebook, LinkedIn, Twitter, etc. A wide selection of journals (inclusive of 9 subjects, more than 200 journals)

Providing 24-hour high-quality service

User-friendly online submission system

Fair and swift peer-review system

Efficient typesetting and proofreading procedure

Display of the result of downloads and visits, as well as the number of cited articles Maximum dissemination of your research work

Submit your manuscript at: http://papersubmission.scirp.org/

Or contact alamt@scirp.org 